\title{
Retrenchment: Extending the Reach of Refinement
}

\author{
M.R. Poppleton ${ }^{\dagger \ddagger} \quad$ R.H. Banach ${ }^{\ddagger}$ \\ †School of Mathematical and Information Sciences, Coventry University, Coventry CV1 5FB, UK \\ ‡Department of Computer Science, Manchester University, Manchester M13 9PL, UK \\ m.r.poppleton@coventry.ac.uk, banachecs.man.ac.uk
}

\begin{abstract}
Discussion of a simple example demonstrates various expressive limitations of the refinement calculus, and suggests a liberalization of refinement, called retrenchment, which will support an analogous formal development calculus. Useful concrete system behaviour can be specified outside the domain of pure refinement, and a case is made for fluidity between I/O and state components across the development step.

A syntax and a formal definition are presented for retrenchment, which has some necessary properties for a formal development calculus: transitivity gives stepwise composition of retrenchments, and monotonicity w.r.t. the specification language constructors gives piecewise construction of retrenchments.
\end{abstract}

\section{Introduction and résumé of $B$}

From early concerns about proving correctness of programs such as Hoare's [19] and Dijkstra's [15], a mature refinement calculus of specifications to programs has developed. This development has been in two strands, the predicate transformer/ lattice-theoretic, e.g. $[4,23,6,16]$ and the relational, e.g. [20, 21]. The first relational proposal for a sound and complete proof method for data refinement was [18]; a modern version of this simulation method appears in [28] in the $\mathrm{Z}$ notation [27].

In this context the term "refinement" has a very precise meaning; according to Back and Butler [5] it is a “...correctness-preserving transformation...between (possibly abstract, non-executable) programs which is transitive, thus supporting stepwise refinement, and is monotonic w.r.t. program constructors, thus supporting piecewise refinement." Relationally, refinement is characterised as a development step requiring the concrete precondition to be weaker than the abstract, and the concrete transition relation to be stronger, or less nondeterministic, than the abstract.
Refinement of specifications on abstract, infinite domains to finite computer-oriented domains poses particular problems [13, 11]. Partial logic approaches have also been proposed [10, 24]. Hayes and Sanders [17] recognise the separation of concerns afforded to the specifier by an abstraction relation between abstract and concrete I/O. Boiten and Derrick [12] propose rules for "I/O refinement", a simple generalisation of Woodcock and Davies' method [28], incorporating abstraction relations for I/O. In the $\mathrm{OO}$ arena, Mikhajlova and Sekerinski's "interface refinement" [22] generalizes "class refinement" in a similar way to which I/O refinement generalizes the classical version in [12].

In this vein we argue that refinement is restrictive in terms over and above those of finiteness and I/O representation. The requirement that every concrete behaviour be a possible abstract one ("operational indistinguishability", or "simulability") is too strong for a finite, discrete computer. Nonsimulable concrete behaviour may be desirable, and its nonsimulability may avoid the need to force implementation constraints into the abstract model. We also argue for a degree of fluidity between state and I/O components across the development step. This leads to retrenchment (introduced in [7]) and its formalisation in a formal calculus. We use a light version of the B notation of Jean-Reymond Abrial [1], which is a descendant of both theoretical strands discussed above. The B-Method is a full-lifecycle formal development method for sequential and reactive systems, supported by two industrial-strength toolkits, Atelier $B$ [2] and $B$-Toolkit [26], and supported by numerous successful industrial applications, e.g. [14].

$\mathrm{B}$ is based on a total correctness theory of programming. Its central construct is the $w p$ predicate transformer: $w p(S, R)$ describes the weakest precondition, or most general before-state from which program $S$ is guaranteed to terminate satisfying postcondition $R$. In $\mathrm{B}, w p$ distributes over conjunction and is monotonic w.r.t. implication. $w p$ does not satisfy Dijkstra's [15] "Law of the Excluded Miracle" (which would require that $w p(S$, false) $\equiv$ false): this allows a notion of feasibility of programs. Programs (in 
general nondeterministic) are written in B using constructors inspired by Dijksta's Guarded Command Language, called the Generalised Substitution Language (GSL). The basic operation is the simple substitution (which is assignment, in procedural programming terms). For replacement of free variable $x$ in formula $R$ by expression $E$ (no free variable in $E$ clashes with any bound variable in $R$ ) we write $w p(x:=E, R)$. The remaining simple constructors of $\mathrm{B}$ are axiomatised as follows (for unbounded choice $z$ is nonfree in $R$; this will be written $z \backslash R)$ :

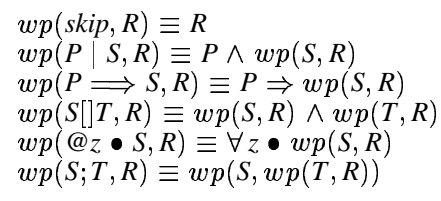
skip
precondition
guard
bounded choice
unbounded choice sequence

WPAxioms

The precondition constructor represents an explicit strengthening of the termination set, guard a strengthening of the feasibility set, bounded choice a demonic nondeterministic choice between two operations, and unbounded choice a universally quantified demonic choice over all operations indexed on some (external) variable.

Any operation $S$ working with a state variable (list) $x$ can be expressed in the following normalised form, where $P$ is a predicate in variable(s) $x, Q$ is a predicate in variables $x$ and $x^{\prime}\left(x^{\prime}\right.$ distinct from $\left.x\right)$ :

$$
S=P \mid @ x^{\prime} \bullet\left(Q \Longrightarrow x:=x^{\prime}\right)
$$

From the axioms, this means that for any predicate $R(x)$

$$
w p(S, R) \equiv P \wedge \forall x^{\prime} \bullet\left(Q \Rightarrow w p\left(x:=x^{\prime}, R\right)\right)
$$

In fact, this decomposition into predicates $P$ and $Q$ is unique (modulo logical equivalence of predicates), and these are called $\operatorname{trm}(S)$ (termination predicate: before-states from which $S$ is guaranteed to terminate) and $\operatorname{prd}_{x}(S)$ (beforeafter transition predicate) respectively. The latter form of this theorem gives a nice interpretation of $S$ as a predicate transformer: from initial state $x, S$ establishes $R$ precisely when $S$ terminates at $x$ and every $x^{\prime}$ reachable from $x$ under $S$ satisfies $R$. These predicates can be explicitly defined:

$$
\begin{aligned}
& \operatorname{trm}(S) \widehat{=} w p(S, \text { true }) \\
& \operatorname{prd}_{x}(S) \widehat{=} \neg w p\left(S, x^{\prime} \neq x\right)
\end{aligned}
$$

A relational model is defined in the obvious way (where $s$ is the set of which the state variable $x$ is a member) for the precondition set, and the before-after relation for $S$ :

$$
\begin{aligned}
& \operatorname{pre}(S) \widehat{=} \quad\{x \mid x \in s \wedge \operatorname{trm}(S)\} \\
& \operatorname{rel}(S) \widehat{=} \quad\left\{x, x^{\prime} \mid\left(x, x^{\prime}\right) \in s \times s \wedge \operatorname{prd}_{x}(S)\right\}
\end{aligned}
$$

The abstract syntax of the GSL is expressed in and complemented by the concrete syntax of the Abstract Machine Notation (AMN), which includes constructs for modular structuring. The unit of modularity is the machine, which contains inter alia a state variable (list), an invariant predicate expressing type and other required state constraints, an initialisation, and a set of operations, which are expressed in

\begin{tabular}{|c|c|c|c|c|}
\hline MACHINE & $M(a)$ & $\begin{array}{l}\text { REFINEMENT } \\
\text { REFINES }\end{array}$ & $\begin{array}{l}N \\
M\end{array}$ & \\
\hline VARIABLES & $u$ & VARIABLES & $v$ & \\
\hline INVARIANT & $I(u)$ & INVARIANT & $J(u, v)$ & \\
\hline INITIALISATION & $X(u)$ & INITIALISATION & $Y(v)$ & \\
\hline OPERATIONS & & OPERATIONS & & \\
\hline$S(u, i, o) \widehat{=}$ & & $T(v, i, o) \widehat{=}$ & & \\
\hline END & & END & & RefSyn \\
\hline
\end{tabular}
terms of state, input and output variables. The following syntax shows an abstract machine and a refinement. The latter is a derivative contruct: its invariant clause provides both local variable type and constraint information, as well as the retrieve relation to the abstract variable.

A sufficient condition for refinement (equivalent to classical forward simulation) is expressed relationally as follows. Two abstract machines $M$ and $N$ are defined on state spaces $u$ and $v$ respectively, with a total relation (the retrieve relation) $r: v \leftrightarrow u$, and a bijection between the operations of $M$ and $N$ (say, every operation $S$ of machine $M$ corresponds to exactly one operation $T$ of $N$ ). If for every such pair $(S, T)$ the following hypotheses hold, then $M$ is refined by $N(\text { written } M \sqsubseteq N)^{1}$ :

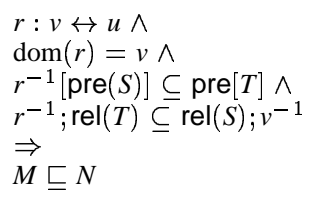

This corresponds to the operation refinement proof obligation (POB) in the following full formulation of the refinement rules in $w p$-form. The obligations are initialisation consistency, operation consistency (given abstract invariant and abstract operation termination, then the operation establishes the invariant), initialisation refinement, and operation refinement (for any concrete step of $T$, there is some abstract step of $S$ that establishes the retrieve relation):

$$
\begin{aligned}
& w p(X, I) \\
& I \wedge \operatorname{trm}(S) \Rightarrow w p(S, I) \\
& w p(Y, \neg w p(X, \neg J)) \\
& I \wedge J \wedge \operatorname{trm}(S) \Rightarrow w p(T, \neg w p(S, \neg J))
\end{aligned}
$$

\section{From refinement to retrenchment}

Let the divine infinite naturals be D-NAT and the mundane finite ones be M-NAT. Consider the following simple abstract machine, modelling subtraction on the divine naturals, and a proposed refinement:

$\begin{array}{cclc}\text { MACHINE } & \text { DivineSub } & \text { REFINEMENT } & \begin{array}{l}\text { MundaneSub } \\ \text { DivineSub }\end{array} \\ & & \text { REFINES } & a a, b b \\ \text { VARIABLES } & a, b & \text { VARIABLES } & \\ \text { INVARIANT } & & \text { INVARIANT } \\ a \in \text { D-NAT } & & a a \in \text { M-NAT } \wedge b b \in \text { M-NAT } \\ \wedge b \in \text { D-NAT } & \wedge(a a<O F \Rightarrow a=a a) \\ & \wedge(a a=O F \Rightarrow a \geq O F)\end{array}$

\footnotetext{
${ }^{1} \mathrm{~A}$ full B definition of refinement is omitted here; see [1]
} 


$$
\begin{aligned}
& \text { OPERATIONS } \\
& \text { DSub } \widehat{=}= \\
& \quad a \geq b \mid a:=a-b
\end{aligned}
$$

END

$$
\begin{aligned}
& \wedge(b b<O F \Rightarrow b=b b) \\
& \wedge(b b=O F \Rightarrow b \geq O F
\end{aligned}
$$

OPERATIONS

resp $\longleftarrow$ MSub $\widehat{=}$

$a a<O F \wedge a a \geq b b$ $\Longrightarrow a a:=\bar{a} a-b b$$$
\text { || resp }:=T
$$

[] $a a \geq O F \vee a a<b b$

$$
\cdots
$$$$
\Longrightarrow \text { resp }:=F
$$

END

The concrete invariant expresses both the concrete type, and the retrieve relation as the obvious total embedding of M-NAT in MundaneSub into D-NAT in DivineSub. The overflow value $O F$ is identified with all large $a$ and $b$ in DivineSub. The abstract precondition is the region $a \geq b$. The concrete operation is clearly of IF..THEN..ELSE form. The concrete precondition is easily calculated by GSL manipulations (using some obvious shorthand). Since both simple substitution operations in $M S u b$ terminate universally, we have

$$
\begin{array}{ll} 
& \operatorname{trm}(G d \Longrightarrow S 1[] \neg G d \Longrightarrow S 2) \\
\equiv \quad & (G d \Rightarrow \operatorname{trm}(S 1)) \wedge(\neg G d \Rightarrow \operatorname{trm}(S 2)) \\
\equiv \quad \text { true } &
\end{array}
$$

This development step cannot universally satisfy OpRef since of course subtraction of an unrepresentably large number from one no smaller cannot be represented: this is a limitation of the finiteness of the concrete model. However, there is a concrete subdomain $D$ where refinement can be achieved. Within $D$ the two concrete variables $a a$ and $b b$ uniquely represent $a$ and $b$ respectively, and the concrete subtraction can be performed. The proof of this "local refinement" is routine. Our first departure from refinement is thus in allowing it to be expressed "locally", in context of other concrete behaviour.

Note that the image of $D$ through the retrieve relation is stronger than the abstract precondition. Its complement covers two situations: (i) Subtraction of large numbers: abstract precondition true, but not concretely representable in a way which will support concrete subtraction, and (ii) Abstract subtraction undefined: abstract preconditon false. Both these situations, where refinement cannot be achieved, have specified (if trivial) concrete behaviour: the output of an error message. The second departure from refinement is demonstrated by the concrete operation: change of operation signature across a refinement step is not allowed. It seems useful to define a mechanism to preserve concrete information when failing to simulate the abstract step.

These proposed features, which depart from standard definitions of refinement, represent a "white-box" design approach to the construction of a development step. This contrasts with refinement's "black-box" approach, where the concrete operation is guaranteed, provided the abstract operation terminates, to provide an operation step indistinguishable from some abstract step. In refinement, the ab- stract model is the best representation of the "real world", and its properties must be preserved at all costs. Abrial [3] believes that it is necessary to know in the abstract model that the "...operation might end up with a funny result (and in that case, with no action performed, or, alternatively, with any modification whatsoever)...”. This view is manifested in [1] by the use of "[] skip" in abstract operations, whereby abstract non-action may be refined by some concrete failure behaviour. The retrenchment view, developed in [7], is that the abstract model should be as straightforward as possible. Any limitations representing it in software on a finite computer should properly be expressed in the concrete model of the software.

This little example suggests how retrenchment, as a liberalization of refinement, might widen expressiveness in the development step. Here is a concrete syntax:

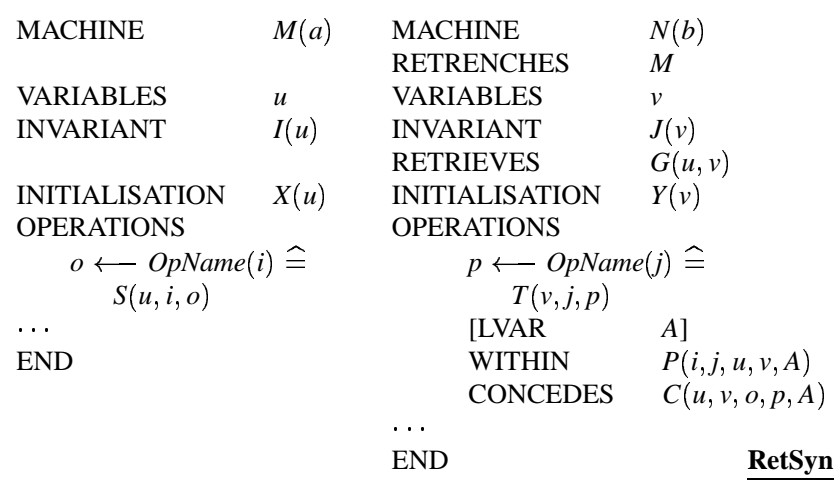

Square brackets indicate optional text. Unlike a refinement, a retrenchment is a machine independent of the $a b-$ stract model. In general either a MACHINE or a REFINEMENT may be retrenched, suggesting an architectural mixture of the two in a series of development steps. The RETRENCHES clause (similarly to REFINES) makes visible the contents of the retrenched machine. We further assume that the name spaces of the retrenched and retrenching machines are disjoint, but admit an injection of (retrenched to retrenching) operation names. An injection allows provision of further, independent operations in the retrenching machine. In fact we overload the term "retrenchment": it refers both to a relationship between machines (indexed by the injection of names), as well as to a relationship between an abstract-concrete operation pair.

In the retrenching machine $N$ we have state variable $v$ under invariant $J(v)$, and the RETRIEVES clause $G(u, v)$. The existence of $N$ as an independent machine requires that its (local) invariant be stated independently of the retrieve relation, unlike in $\mathrm{B}$, where the two take the form of a joint predicate.

In liberalizing refinement we propose that the relationship between concrete and abstract state is fundamentally different before and after the operation. We model this by 
distinguishing between a strengthened before-relation between abstract and concrete states, and a weakened afterrelation. Thus the syntax of the concrete operation OpName in $N$ is precisely as in $\mathrm{B}$, with the addition of the ramification, a syntactic enclosure of the operation. Before, the relationship is constrained (precondition is strengthened) by the WITHIN constraint $P(u, v, i, j, A)$ which may change the balance of components between input and state, and may further define an optional "memory" variable LVAR $A$ for reference in the after-state. The after-relation is weakened (postcondition is weakened) by the CONCEDES clause (the concession) $C(u, v, o, p, A)$, which specifies what the operation guarantees to achieve (in terms of after-state, output and logical variable $A$ ) if it cannot maintain the retrieve relation $G$.

The retrenchment concept is defined by its proof obligations. The initialisation POB Init will be as for refinement, guaranteeing a joint starting state satisfying $G(u, v)$. Retrenchment of operations is defined by the following proof obligation (analogous to OpRef ): concrete operation $T$ retrenches abstract operation $S$ with respect to constraint $P$ and concession $C$. For shorthand we write $S \lesssim_{G, P, C, A} T$, or simply $S \lesssim T$.

$$
\begin{gathered}
I(u) \wedge G(u, v) \wedge J(v) \wedge \operatorname{trm}(T(v, j, p)) \wedge P(i, j, u, v, A) \\
\Rightarrow \operatorname{trm}(S(u, i, o)) \wedge w p(T(v, j, p), \neg w p(S(u, i, o), \\
\neg(G(u, v) \vee C(u, v, o, p, A))))
\end{gathered}
$$

$\underline{\text { RetWP }}$

This definition is justified by comparison with the refinement proof obligation. In refinement, when the abstract operation terminates, so does the concrete one. In retrenchment this relationship is inverted. The retrenching machine can in general simulate the abstract machine only for some before-states. Thus we strengthen the precondition in retrenchment, conjoining the WITHIN constraint $P$ into the proof obligation hypotheses. This restricts the combinations of before-state and inputs (over and above those specified by the RETRIEVES clause) where the concrete operation (viewed as a retrenchment) is meaningful and will terminate.

In refinement, "operational indistinguishability" of the refined operation is the central issue. Retrenchment is weaker: the dynamic behaviour of the operation is guaranteed to achieve $(G \vee C)$, that is, either the RETRIEVES clause or the CONCEDES clause. The concession is defined in terms of abstract and concrete after-state and output, as well as the logical "memory" variable $A$. We also observe from the refinement $\mathrm{POB}$ OpRef that we have preserved the familiar shape of the consequent, i.e. $\forall$ ConcOp $\exists A b s O p \bullet$ $(G \vee C)$. That is, for every concrete transition there exists some abstract transition that either achieves the retrieve relation or at least meets the concession. There are a number of benefits to this shape.

A statement is made about every concrete step; this enhances the quality of the concrete description, ultimately the one more faithful to the final system.

Every concrete step is considered as to whether it is (i) excluded from consideration because either $G, P$ or $\operatorname{trm}(T)$ are not satisfied, (ii) included but can only achieve the concession $C$, or (iii) included and will achieve either $G$ or $C$, or (iv) included and achieves $G$. The latter is refinement-like behaviour. In general, unlike in the example, these cases will not be separable a priori.

We complete this section by expressing the concrete subtraction operation $M S u b$ as a retrenchment:

$$
\begin{aligned}
& \text { MACHINE MundaneSub1 } \\
& \text { RETRENCHES DivineSub } \\
& \text { VARIABLES } a a \\
& \text { INVARIANT } a a \in \text { M-NAT } \\
& \text { RETRIEVES } \quad(a a<O F \Rightarrow a=a a) \wedge(a a=O F \Rightarrow a \geq O F) \\
& \text { OPERATIONS } \\
& r e s p \longleftarrow \operatorname{MSub}(b b) \widehat{=} b b \in \mathrm{M}-\mathrm{NAT} \\
& (a a<O F \wedge a a \geq b b \Longrightarrow a a:=a a-b b \| \text { resp }:=T \\
& \text { [] } a a \geq O F \vee a a<b b \Longrightarrow \text { resp }:=F \text { ) } \\
& \text { LVAR } \\
& \text { WITHIN } \quad A A=a a \wedge(b b<O F \Rightarrow b=b b) \\
& \text { CONCEDES } \quad(\text { resp }=T \Rightarrow a=a a) \\
& \wedge(\text { resp }=F \Rightarrow a a=A A)
\end{aligned}
$$

END

The example, albeit trivial, usefully demonstrates some features of retrenchment. $b b$ moves from state to input, and thus is absent from the RETRIEVES relation and the concrete local INVARIANT. The concrete precondition gives the type of $b b$. It is related to its abstract state counterpart $b$ through the WITHIN clause. The WITHIN clause also uses local "memory" variable $A A$ to record before-state $a a$, in order establish that the latter's value remains unchanged where the subtraction cannot be performed. Here the CONCEDES clause is quite expressive, describing the after-state situation $(r e s p=T)$ under which the RETRIEVES clause will be maintained, as well as the "concession" option (here, keeping the value of $a a$ unchanged). Next consider the simplified retrenchment $\mathrm{POB}$ for the example:

$$
\begin{aligned}
& a \in \mathrm{D}-\mathrm{NAT} \wedge b \in \mathrm{D}-\mathrm{NAT} \wedge a a \in \mathrm{M} \text {-NAT } \\
& \wedge(a a<O F \Rightarrow a=a a) \\
& \wedge(a a=O F \Rightarrow a \geq O F) \\
& \wedge A A=a a \wedge(b<O F \Rightarrow b=b b) \\
& \wedge b b \in \mathrm{M}-\mathrm{NAT} \\
& \Rightarrow a \geq b \\
& \wedge w p(a a<O F \wedge a a \geq b b \\
& \Longrightarrow a a:=a a-b b \| \text { resp }:=T \\
& \text { [] } a a \geq O F \vee a a<b b \Longrightarrow \text { resp }:=F, \\
& \neg w p(a \geq b \mid a:=a-b \text {, } \\
& \neg(a=a a \vee((\text { resp }=T \Rightarrow a=a a) \\
& \wedge(\operatorname{resp}=F \Rightarrow a a=A A))))
\end{aligned}
$$

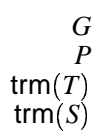

Applying WPAxioms, the consequent above expands to a conjunction of three subgoals:

$$
\begin{aligned}
a \geq b & \\
\wedge(b b \in \mathrm{M}-\mathrm{NAT} \wedge a & \wedge a \mathrm{OF} \wedge a a \geq b b \\
\Rightarrow w p(a a & :=a a-b b \| \text { resp }:=T, \\
\neg w p(a \geq b \mid a & :=a-b, \\
& \neg(a=a a \vee((\text { resp }=T \Rightarrow a=a a)) \\
& \wedge(\operatorname{resp}=F \Rightarrow a a=A A)))))
\end{aligned}
$$




$$
\begin{aligned}
& \wedge(b b \notin \text { M-NAT } \vee a a \geq O F \vee a a<b b \\
& \Rightarrow w p(\operatorname{resp}::=F, \\
& \neg w p(a \geq b \mid a:=a-b, \\
& \neg(a=a a \vee((\text { resp }=T \Rightarrow a=a a) \\
&\wedge(\operatorname{resp}=F \Rightarrow a a=A A)))))
\end{aligned}
$$

Clearly there is insufficient given hypothesis to infer abstract termination $a \geq b$. This can easily be resolved by conjoining this clause as an assumption into the WITHIN constraint. However it is illustrative that we do not do this in the example. Such an assumption precludes description of concrete behaviour outside the abstract precondition, where such description is part of the motivation for retrenchment.

In the second subgoal, abstract termination follows from the extra information in the guard about $a a$ and $b b$. This is the only situation where retrenchment can reduce to refinement. In the example, this subgoal in fact can be strengthened to a refinement (modulo $\mathrm{I} / \mathrm{O} /$ state variable migration). We call this special case inverted modulated refinement and examine this notion further in [9]. It is inverted in the sense of inverting the relationship of the preconditions in the refinement POB OpRef , and modulated in permitting a migration between $\mathrm{I} / \mathrm{O}$ and state across the development step.

The third subgoal covers both unrepresentable abstract state, as well as the region where the abstract operation is undefined. This represents the "exception" behaviour that contributes to a weak concrete precondition, breaching RetWP. This is not a problem; clearly the significant theoretical development for retrenchment is within the definition, i.e. within the abstract precondition which scopes the domain of possible local refining behaviour.

This split nicely demonstrates a white-box design style: we can disjunctively split (not always a priori) a weak, or vacuously true concrete precondition, into regions where we can guarantee (modulated) refinement, guarantee either refinement or some useful concession, or guarantee only the concession clause. We build the concrete model as onion layers of increasing complexity on top of the abstract model. In general the case split will be applicationdependent, less crisp than in the example, and have finer, more elaborate structure. Complex POB's will emerge which are nonetheless first-order and should be tractable by mechanised theorem-provers.

The retrenchment notion was inspired by the failure of refinement to express useful development steps for systems with continuous variables and properties: finite data types must approximate the real numbers, giving rise to the problems of precision decay examined in Numerical Analysis. Such a development could be expressed in terms of notions either of "degrading" invariants, or weakening concessions in retrenchment.

Finally note that retrenchment liberalizes refinement: every refinement yields a retrenchment, using abstract precondition as WITHIN constraint and a false concession.

\section{A relational theory of retrenchment}

We give an overview of a relational model for retrenchment; a full development is in preparation [25].

In section $1, \mathrm{~B}$ relational semantics was expressed in terms of state variables, with I/O afforded no special status. The more fluid world of retrenchment requires that $\mathrm{I} / \mathrm{O}$ variables become "first-class citizens". This is achieved by adding appropriate dimensionality to the semantic model; that is, an operation $S$ with state $u$, input $i$, output $o$ is described in terms of a heterogeneous $w p$ definition:

$$
w p\left(S,,_{-}\right):(\underline{u} \times \underline{o} \rightarrow \mathbb{B}) \rightarrow(\underline{u} \times \underline{i} \rightarrow \mathbb{B})
$$

The normal form theorem Norm is expressible in GSL, extending the definitions of trm and prd in an obvious way. Its $w p$-semantics are given, for operation $S(u, i, o)$ and predicate $R(u, o)$, by

$$
\begin{aligned}
& w p(S(u, i, o), R(u, o)) \widehat{=} \operatorname{trm}(S)(u, i) \\
& \wedge \forall u^{\prime}, o^{\prime} \bullet\left(\operatorname{prd}_{u}(S)\left(u, i, u^{\prime}, o^{\prime}\right) \Rightarrow\left[u, o:=u^{\prime}, o^{\prime}\right] R(u, o)\right)
\end{aligned}
$$

By simple expansion, the defining proof obligation for retrenchment RetWP can be shown equivalent to the following more explicit conjunction of termination and transition conditions (free in $u, v, i, j, A)$ :

$$
\begin{aligned}
& (I(u) \wedge G(u, v) \wedge J(v) \wedge \operatorname{trm}(T(v, j, p)) \wedge P(i, j, u, v, A) \\
& \Rightarrow \operatorname{trm}(S(u, i, o))) \\
& (I(u) \wedge G(u, v) \wedge J(v) \wedge \operatorname{trm}(T(v, j, p)) \wedge P(i, j, u, v, A) \\
& \Rightarrow \forall v^{\prime}, p^{\prime} \bullet \operatorname{prd}_{v}(T) \\
& \Rightarrow \exists u^{\prime}, o^{\prime} \bullet \operatorname{prd}_{u}(S) \\
& \left.\left.\left.\wedge\left(G\left(u^{\prime}, v^{\prime}\right) \vee C\left(u^{\prime}, v^{\prime}, o^{\prime}, p^{\prime}, A\right)\right)\right)\right)\right)
\end{aligned}
$$

Relationally speaking, the abstract operation relates beforestate and input to after-state and output. The concrete operation (now including the "memory variable" $A$ ) relates two triples: (before-state, input, variable) to (after-state, output, variable). That is,

$$
\begin{aligned}
& \operatorname{rel}(S): \underline{u} \times \underline{i} \leftrightarrow \underline{u} \times \underline{o} \\
& \operatorname{rel}(T) \| \operatorname{id}(\bar{A}): \underline{v} \times \underline{j} \times \underline{A} \leftrightarrow \underline{v} \times \underline{p} \times \underline{A}
\end{aligned}
$$

The following four definitions give the relational model for retrenchment, which will be seen to be equivalent to the predicate definition $\underline{\text { Ret }}$. In place of the retrieve relation, define two retrenchment relations, respectively defining the strengthened before-state, and weakened after-state situations. Call these $r_{j}$ and $r_{p}$ respectively, naming subscripts after concrete input and output.

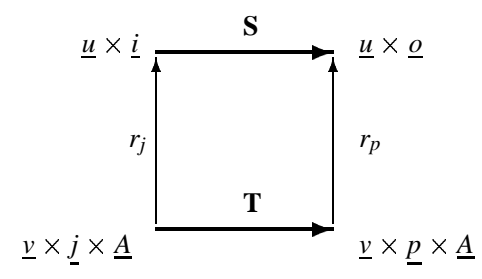

Figure 1. Retrenchment: a relational model 
$r_{j}=\{(v, j, A),(u, i) \mid I(u) \wedge G(u, v) \wedge J(v) \wedge P(i, j, u, v, A)\}$

$r_{p}=\{(v, p, A),(u, o) \mid J(v) \wedge(G(u, v) \vee C(u, v, o, p, A))\}$

Ret1R,Ret2R

The termination condition for retrenchment, analogous to that in $\underline{\mathbf{R e f R}}$, represents precondition strengthening. A stronger definition satisfying $B$ semantics reduces easily to

$$
r_{j}[\operatorname{pre}(T) \times \underline{A}] \subseteq \operatorname{pre}(S)
$$

$\underline{\text { Ret3R }}$

The transition condition also mimics refinement analo-

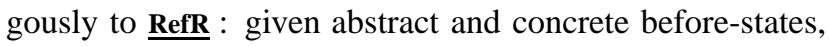
for every terminating concrete step there is at least one abstract step that establishes $r_{p}$, i.e. either the RETRIEVES clause $G$ or the concession $C$. Again a stronger definition reduces to:

$$
r_{j}^{-1} ;(\operatorname{pre}(T) \triangleleft \operatorname{rel}(T) \| \operatorname{id}(A)) \subseteq \operatorname{rel}(S) ; r_{p}^{-1}
$$

$\underline{\text { Ret4R }}$

The following theorem states the equivalence of the predicate and relational models:

\section{Theorem Equiv}

$\underline{\text { Ret }} \wedge \underline{\text { OpCons }} \vdash \underline{\text { Ret3R }} \wedge \underline{\text { Ret4R }}$

$\underline{\operatorname{Ret} 3 R} \wedge \underline{\text { Ret4R }} \vdash \underline{\text { Ret }}$

Note the asymmetry of the result: the relational model ( $\underline{\operatorname{Ret} 3 \mathbf{R}}, \underline{\mathbf{R e t} 4 \mathbf{R}}$ ) is stronger than the predicate model $\underline{\text { Ret }}$, whereas the concrete operation consistency condition is required to infer the relational from the predicate model. This is because the relational model requires concrete invariant $J$ to be invariant in the retrenchment, while the predicate model does not.

Next we show that retrenchments compose. That is, the retrenchment ordering on operations is transitive, as is required for retrenchment to be a stepwise-composable development approach. Although the proof is essentially relational, it is necessary to demonstrate that the composed before- and after-retrieve relations correspond to a syntactically well-formed retrenchment; this is straightforward and is not elaborated here. We state a slightly weakened result as a proof obligation in the $w p$-style, which can be regarded as a definition of composed retrenchment.

Firstly a lemma is required, which combines the termination and transition conditions $\underline{\mathbf{R e t} 3 \mathbf{R}}$ and $\underline{\mathbf{R e t} 4 \mathbf{R}}$ in a useful and intuitive way:

$$
\begin{aligned}
& \underline{\operatorname{Ret} 3 R} \wedge \underline{\operatorname{Ret} 4 \mathbf{R}} \\
& \overline{\vdash r_{j}^{-1}} ;(\operatorname{pre}(T) \triangleleft \operatorname{rel}(T) \| \operatorname{id}(A)) \subseteq(\operatorname{pre}(S) \triangleleft \operatorname{rel}(S)) ; r_{p}^{-1} \\
& \text { Comp }
\end{aligned}
$$

Composition of retrenchment has been shown directly [7] by combining the component GS-form retrenchment obligations into the required shape. We will show composition relationally, by demonstrating that the two conditions $\underline{\text { Ret3R }}$ and $\underline{\text { Ret4R }}$ compose as expected. Figure 2 shows the operations and retrenchment relations involved.

Assume as before (RetSyn ) that machine $N$ RETRENCHES $M$, and further that machine $O$ RETRENCHES $N$. We define machine $O$ syntactically as a "lexicographic increment" on $N$, systematically replacing occurrences of $N, b, M, v, J, G, Y, p, j, T, A, P, C$ in $N$ by

$O, c, N, w, K, H, Z, q, k, U, B, Q, D$, respectively. Thus $O$ has INVARIANT $K$, RETRIEVES $H$, operation $U$, logical variable $B$, WITHIN $Q$ and concession $D$. Operation $S$ in machine $M$ is retrenched by operation $T$ in machine $N$, which is in turn retrenched by operation $U$ in machine $O$.

Relationally, $S$ is retrenched by $T$, mediated by beforeand after-retrieve relations $\left(r_{j}, r_{p}\right)$. This means that $r_{j}$ and $r_{p}$ encapsulate the syntactic definition of the retrenchment as follows:

$$
\begin{aligned}
& r_{j}=\{(v, j, A),(u, i) \mid I(u) \wedge \\
& G(u, v) \wedge \\
& J(v) \wedge \\
&P(i, j, u, v, A)\} \\
& r_{p}=\{(v, p, A),(u, o) \mid J(v) \\
&(G(u, v) \vee \\
&C(u, v, o, p, A))\}
\end{aligned}
$$

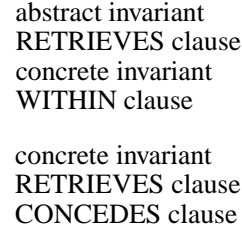

That is, the defining predicates for $r_{j}$ and $r_{p}$ are conjunctions of clauses free in variable lists that suitably identify these clauses with those of the syntactic definition. Similarly, $T$ is retrenched by $U$, mediated by $\left(s_{k}, s_{q}\right)$, which are defined again by "lexicographic increment".

We will prove that $S$ in $M$ is retrenched by $U$ in $O$, mediated by the composed relations $t_{k}=s_{k} ; r_{j}$ and $t_{q}=s_{q} ; r_{p}$, with the same interpretation as for the component relations above. It is simple to check that the defining predicates of $t_{k}$ and $t_{q}$ have the shape required to specify a syntactically well-formed candidate retrenchment.

Theorem RetCompR : Assume $S$ in $M$ is retrenched by $T$ in $N$, mediated by $\left(r_{j}, r_{p}\right)$, and further that $T$ in $N$ is retrenched by $U$ in $O$, mediated by $\left(s_{k}, s_{q}\right)$. Assume syntactic definitions for these relations as given above (omit the logical variables $A, B$ for simplicity). Then $S$ is retrenched by $U$, mediated by $\left(t_{k}=s_{k} ; r_{j}, t_{q}=s_{q} ; r_{p}\right)$. That is,

$$
\begin{aligned}
& t_{k}[\operatorname{pre}(U)] \subseteq \operatorname{pre}(S) \\
& t_{k}^{-1} ;(\operatorname{pre}(U) \triangleleft \operatorname{rel}(U)) \subseteq \operatorname{rel}(S) ; t_{q}^{-1}
\end{aligned}
$$

\section{Proof}

Consider the composition of transition conditions:

Given

$$
\begin{aligned}
& r_{j}^{-1} ;(\operatorname{pre}(T) \triangleleft \operatorname{rel}(T)) \subseteq \operatorname{rel}(S) ; r_{p}^{-1} \\
& s_{k}^{-1} ;(\operatorname{pre}(U) \triangleleft \operatorname{rel}(U)) \subseteq \operatorname{rel}(T) ; s_{q}^{-1}
\end{aligned}
$$

$\mathrm{H} 2$

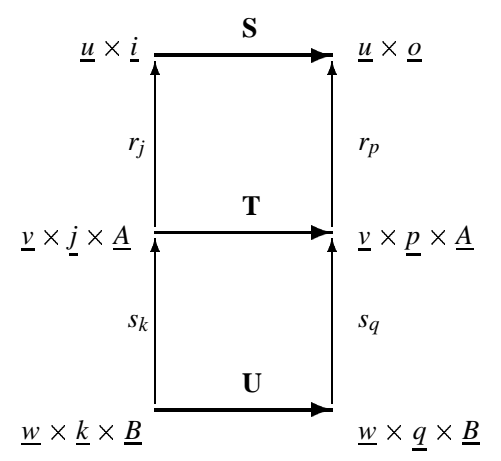

Figure 2. Composition of retrenchments 


$$
\begin{aligned}
& r_{j}[\operatorname{pre}(T)] \subseteq \operatorname{pre}(S) \\
& s_{k}[\operatorname{pre}(U)] \subseteq \operatorname{pre}(T) \\
& \text { Infer by Comp } \\
& s_{k}^{-1} ;(\operatorname{pre}(U) \triangleleft \operatorname{rel}(U)) \subseteq(\operatorname{pre}(T) \triangleleft \operatorname{rel}(T)) ; s_{q}^{-1} \\
& \text { We must now show that } \\
& t_{k}^{-1} ;(\operatorname{pre}(U) \triangleleft \operatorname{rel}(U)) \subseteq \operatorname{rel}(S) ; t_{q}^{-1} \\
& \text { That is, by associativity of ; } \\
& r_{j}^{-1} ; s_{k}^{-1} ;(\operatorname{pre}(U) \triangleleft \operatorname{rel}(U)) \subseteq \operatorname{rel}(S) ; r_{p}^{-1} ; s_{q}^{-1} \\
& \text { Now observe that, by monotonicity of ; over } \subseteq \\
& r_{j}^{-1} ; s_{k}^{-1} ;(\operatorname{pre}(U) \triangleleft \operatorname{rel}(U)) \\
& \subseteq \quad r_{j}^{-1} ;(\operatorname{pre}(T) \triangleleft \operatorname{rel}(T)) ; s_{q}^{-1} \quad \text { by D1 } \\
& \subseteq \operatorname{rel}(S) ; r_{p}^{-1} ; s_{q}^{-1} \quad \text { by } \mathrm{H} 1 \\
& s_{k}[\operatorname{pre}(U)] \subseteq \operatorname{pre}(T) \\
& \text { We must show that } \\
& t_{k}[\operatorname{pre}(U)] \subseteq \operatorname{pre}(S) \\
& L H S=\left(s_{k} ; r_{j}\right)[\operatorname{pre}(U)]
\end{aligned}
$$

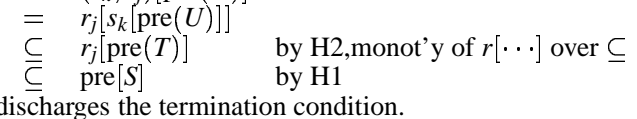
QED

Note that the proof of existence of a composed retrenchment is purely relational, given relational definitions of the component retrenchments. A purely relational theory of refinement exists [28]; this work represents part of a relational theory for retrenchment.

A POB for the composed retrenchment can now be written down by expanding $\left(t_{k}, t_{q}\right)$. It is weakened slightly to remove repeated occurrences of intermediate invariant $J(v)$ in the concession, giving

\section{Theorem RetCompWP}

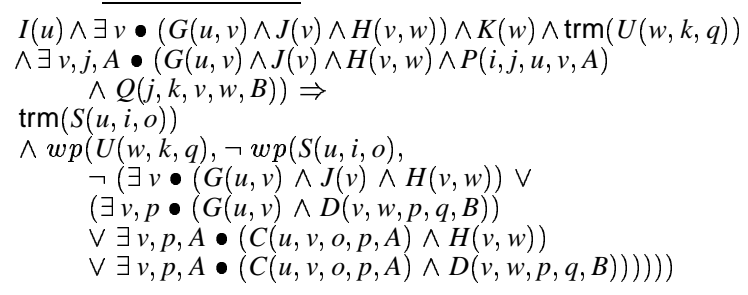

The result is intuitively satisfying. The RETRIEVES clause combines component RETRIEVES clauses and intermediate invariant. The WITHIN clause combines all component before-state RETRIEVES and WITHIN constraints. The concession is a cross-product of component RETRIEVES clauses and concessions.

An associativity theorem can be proved routinely:

Theorem RetAssoc : Weak vertical composition of retrenchments is associative, modulo cancellation of all intermediate-state invariant terms.

\section{Monotonicity properties}

It is straightforward to show that the simple GSL constructors are monotonic w.r.t. the retrenchment ordering, as is required in order that retrenchment be a piecewise development step (see [25]). Monotonicity results are stated for the four primitive GSL constructors.

Theorem RetMono : Retrenchment is monotonic w.r.t. the precondition, guard, bounded and unbounded choice constructors of B.

Case | : Assume $S(u, i, o) \lesssim_{G, P, C, A} T(v, j, p)$. Assume $Q$ is a predicate either on a common input variable or some shared external variable unaffected by either operation. Provided $u, v, o, p$ are nonfree in $Q$, then

$$
(Q \mid S) \lesssim_{G, P, C, A}(Q \mid T)
$$

Case $\Longrightarrow$ : Assume $S(u, i, o) \lesssim_{G, P, C, A} T(v, j, p)$. Assume $Q$ as for case | above. Then

$$
(Q \Longrightarrow S) \lesssim_{G, P, C, A}(Q \Longrightarrow T)
$$

Case [ : Assume $S 1(u, i, o) \lesssim_{G, P 1, C 1, A} T 1(v, j, p)$ and $S 2(u, i, o) \lesssim_{G, P 2, C 2, A} T 2(v, j, p)$. Then

$$
S 1[] S 2 \lesssim_{G, P 1 \wedge P 2, C 1 \vee C 2, A} T 1[] T 2
$$

Case @ : Now assume that $S(u, i, o, x) \lesssim_{G, P, C, A}$ $T(v, j, p, x)$ for some fresh free external variable $x$ (distinct from $u, i, v, j, p, A)$. Then

$$
@ x \bullet S \lesssim_{G, P, C, A}^{@ x} \bullet T
$$

\section{Sequence}

Returning briefly to refinement, in [1] a monotonicity result is stated for refinement w.r.t. operation sequence (called “;”). The simulation property (given a collection of operation refinements, it follows that an arbitrary abstract operation sequence is refined by the corresponding concrete operation sequence) follows implicitly from the associativity of ";". Thus a full retrenchment calculus requires that, given a collection of operation retrenchments, an arbitrary abstract operation sequence should, under suitable assumptions (composed WITHIN constraint) be retrenched by the corresponding concrete operation sequence. The argument is analogous to that for refinement, if rather more intricate; after extending the standard definition of "; ", we give a monotonicity result for a sequence of retrenchments. We state the result here, which is not associative in this form. Its proof and a discussion of associativity appear in [25].

The sequence of retrenchments result has three conjuncts in its WITHIN clause. The first requires that the first-step WITHIN constraint $P 1$ be witnessable for some logical variable. The second, satisfying intuition, requires the first-step retrenchment to be able to establish both the RETRIEVES clause and the second-step WITHIN clause P2: the second step must be guaranteed to start before sequenced retrenchment can be established. The third conjunct is a weakened termination hypothesis, which means that the sequenced termination result (that is, inference of abstract sequenced termination) is nontrivial. The sequenced concession is 
identical to the second-step concession. The result is, using syntax as per RetWP :

\section{Theorem RetSeq}

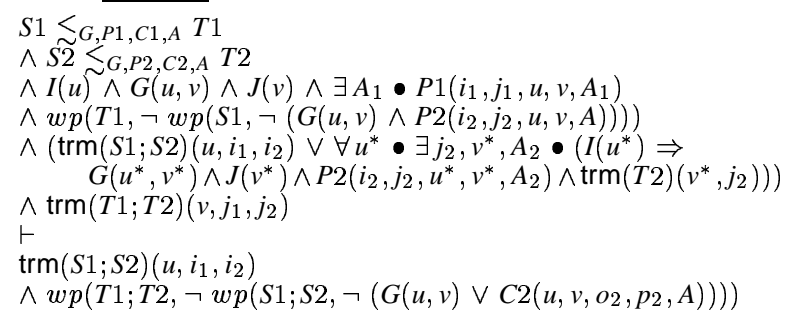

Special cases of retrenchment have been considered, yielding strengthened results for simulation in $[9,8]$.

\section{Conclusion}

In this paper we have presented an extended argument for a liberalized notion of refinement, i.e. retrenchment. Transitivity of this development relation means that it is composable stepwise, and the monotonicity results mean that retrenchments can be constructed piecewise.

It is interesting to observe that Boiten and Derrick's I/O refinement [12] appears to be expressible as a special-case retrenchment. Their abstraction relations for input and output are expressible as an input-only WITHIN constraint and an output-only concession. Our concession is disjunctive in the postcondition; we have examined the possibility of establishing conjunctive postcondition information in [9], in a variation called sharp retrenchment. This variation is a crisper vehicle for describing an I/O refinement.

\section{References}

[1] J.-R. Abrial. The B-Book: Assigning Programs to Meanings. Cambridge University Press, 1996.

[2] J.-R. Abrial. http://www.atelierb.societe.com/index_uk.html, 1998. Atelier-B.

[3] J.-R. Abrial. private communication. 1999.

[4] R. Back. On correct refinement of programs. J. Comp. Sys. Sci., 23:49-68, 1981.

[5] R. Back and M. Butler. Fusion and simultaneous execution in the refinement calculus. Acta Informatica, 1998.

[6] R. J. R. Back and J. von Wright. Refinement calculus part I: Sequential nondeterministic programs. In de Roever and Rozenberg, editors, Proc. REX Workshop, Stepwise Refinement of Distributed Systems, volume 430 of LNCS, pages 42-66. Springer, 1989.

[7] R. Banach and M. Poppleton. Retrenchment: An engineering variation on refinement. In D. Bert, editor, Proc. B'98:Recent Advances in the Development and Use of the B Method, volume 1393 of LNCS, Montpellier, France, April 1998. Springer.

[8] R. Banach and M. Poppleton. Retrenchment and punctured simulation. In K. Araki, A. Galloway, and K. Taguchi, edi- tors, Proc. IFM'99:Integrated Formal Methods 1999, pages 457-476, University of York, June 1999. Springer.

[9] R. Banach and M. Poppleton. Sharp retrenchment, modulated refinement and punctured simulation. Formal Aspects of Computing, 1999. submitted for publication.

[10] H. Barringer, J. Cheng, and C. Jones. A logic covering undefinedness in program proofs. Acta Informatica, 21:251-269, 1984.

[11] A. Blikle. The clean termination of iterative programs. Acta Informatica, 16:199-217, 1981.

[12] E. Boiten and J. Derrick. IO-refinement in Z. In Third BCSFACS Northern Formal Methods Workshop, Ilkley, U.K., Aug 1998.

[13] D. Coleman and J. Hughes. The clean termination of pascal programs. Acta Informatica, 11:195-210, 1979.

[14] B. Dehbonei and F. Mejia. Formal development of safety-critical software systems in railway signalling. In M. Hinchey and J. Bowen, editors, Applications of Formal Methods, chapter 10, pages 227-252. Prentice-Hall, 1995.

[15] E. Dijkstra. A Discipline of Programming. Prentice-Hall, 1976.

[16] P. Gardiner and C. Morgan. A single complete rule for data refinement. Formal Aspects of Computing, 5:367-382, 1993.

[17] I. J. Hayes and J. W. Sanders. Specification by interface separation. Formal Aspects of Computing, 7(4):430-439, 1995.

[18] J. He, C. Hoare, and J. Sanders. Data refinement refined. In B. Robinet and R. Wilhelm, editors, ESOP86: European Symposium on Programming, volume 213 of LNCS. Springer, 1986.

[19] C. Hoare. An axiomatic basis for computer programming. Communications of the ACM, 12(10):576-583, 1969.

[20] C. Hoare and J. He. The weakest prespecification. Information Processing Letters, 24:127-132, 1987.

[21] C. Hoare, J. He, and J. Sanders. Prespecification in data refinement. Information Processing Letters, 25:71-76, 1987.

[22] A. Mikhajlova and E. Sekerinski. Class refinement and interface refinement in object-oriented programs. In J. Fitzgerald, C. Jones, and P. Lucas, editors, FME'97:Industrial Applications and Stengthened Foundations of Formal Methods, volume 1313 of LNCS, pages 82-101, Graz, Austria, 1997. Springer.

[23] J. M. Morris. A theoretical basis for stepwise refinement and the programming calculus. Science of Computer Programming, 9:287-306, 1994.

[24] O. Owe. Partial logics reconsidered: A conservative approach. Formal Aspects of Computing, 3:1-16, 1993.

[25] M. Poppleton and R. Banach. Retrenchment: a relational theory. 1999. in preparation.

[26] I. Sørensen. http://www.b-core.com/, 1998. B-Core(UK) Ltd.

[27] J. M. Spivey. The $Z$ Notation: A Reference Manual. Prentice-Hall, second edition, 1993.

[28] J. Woodcock and J. Davies. Using Z: Specification, Refinement and Proof. Prentice-Hall, 1996. 